\title{
The impact of dynamic RTS Threshold adjustment for IEEE 802.11 MAC protocol
}

\author{
Mostafa Mjidi, Debasish Chakraborty, Naoki Nakamura and Norio Shiratori \\ ${ }^{a}$ Research Institute of Electrical Communication, Graduate School of Information Sciences, Tohoku \\ University, 2-1-1 Katahira, Aoba-ku, Sendai 980-8577, Japan \\ Tel.: +81 22217 5454; E-mail: \{mostafa,deba,norio\}@shiratori.riec.tohoku.ac.jp \\ ${ }^{\mathrm{b}}$ School of Medicine, Tohoku University, 2-1 Seiryoumachi, Aoba-ku, Sendai, 980-8575, Japan \\ Tel.: +8122717 8024; E-mail: nakamura@med.tohoku.ac.jp
}

\begin{abstract}
In recent years, wireless technologies and application received great attention. The Medium Access Control (MAC) protocol is the main element that determines the efficiency in sharing the limited communication bandwidth of the wireless channel in wireless local area networks (WLANs). IEEE 802.11 introduced the optional RTS/CTS handshaking mechanism to address the hidden terminal problem as well as to reduces the chance of collision in case of higher node density and traffic. RTS Threshold (RT) determines when RTS/CTS mechanism should be used and proved to be an important parameter for performance characteristics in data transmission. We first investigate to find a meaningful threshold value according to the network situation and determine the impact of using or disengaging the RTS/CTS optional mechanism and dynamically adjust the RTS Threshold to maximize data transmission. The results show a significant improvement over existing CSMA/CA and RTS/CTS schemes. Our adaptive scheme performed even better when data rate increases. We verify our proposed scheme both analytically and with extensive network simulation using ns-2.
\end{abstract}

Keywords: IEEE 802.11, MAC, RTS/CTS Threshold

\section{Introduction}

Wireless communication providing users with network connectivity without being restricted by a wired network. Ad hoc wireless local area network is a collection of mobile hosts, forming a temporary network without the aid of any pre-established infrastructure or centralized administration. As a result, where conventional networking is difficult or not economic wireless applications are preferred for its convenience and lower price. IEEE 802.11 working group has provided detail specification of MAC and physical layer for WLAN [1]. The performance of mobile ad hoc networks depends on efficient channel sharing of wireless network. Among these, IEEE 802.11 MAC is clearly the most accepted and widely used one at present. The sharing of channel is controlled by the Medium Access Control (MAC) protocol [3]. Since any transmission in a WLAN is relies on a common and open radio medium, the MAC protocol in WLAN would be more important than in conventional wired networks.

The IEEE 802.11 WLAN standard includes a basic distributed coordination function (DCF) and a point coordination function (PCF) which is a centralized MAC protocol that supports collision free time bounded services. In order to control contentions, carrier sense based random-access multiple access algorithms are used. IEEE 802.11 uses the standard transmission scheme of Carrier Sensing Multiple Access with Collision Avoidance (CSMA/CA), which can operate efficiently. Depending on 
the geographical positioning of the nodes, hidden and exposed terminal problem can occur, because in wireless networks, interference is location based. Resolving hidden terminal problem becomes one of the major design considerations of MAC protocol used in both wireless LANs and mobile ad hoc networks (MANETs). To partly overcome the hidden terminal problem, a four way handshaking scheme (RTS/CTS) of channel reservation was introduced as an option in IEEE 802.11 MAC protocol. The RTS/CTS mechanism reserves the channel for transmissions involving larger data packets, with the desired effect less bandwidth would be wasted when collision occurs. On the other hand, when an extremely short packet needs to be sent, we might not benefit, and on the contrary even consume extra bandwidth from RTS/CTS mechanism. Here, an RTS Threshold (RT) acts as a switch between the two schemes and its a manageable parameter used to determine when an RTS/CTS should be precede a data packet. RT is not specified by IEEE 802.11 standard and has to be managed separately by each node.

In this paper, we proposed an algorithm to dynamically adjust the RT depending on the variations of network density and traffic based on short-term storage of packet delivery ratios. If the ratio drops below a certain threshold value, which is determined by investigation through simulation, RTS/CTS handshaking is used to avoid collision, else the packets is sent directly using basic scheme. The evaluation is done both analytically as well as by computer simulation using ns- 2 and the results show that they are corroborating each other. The main advantages of our approach is the simplicity and high accuracy rate. Besides, it relies only on success rate of packet delivery, irrespective of the network size. The adaptive adjustment of RT assures the balance between higher collision penalty and better channel utilization.

The remainder of this paper is organized as follows: In Section 2, we summarized the background and the related works. The numerical comparison is given in Section 3. In Section 4 we introduce our proposed scheme. Both analytical and simulation based evaluation results are shown in Section 5. Finally we conclude the work and present future works in Section 6.

\section{Background: Distributed Coordination Function}

We will briefly describe the Distributed Coordinated Function (DCF), as we focus on it in this article. A complete presentation can be found in [1].

MAC protocol used in DCF is CSMA/CA, which consists of two types of carrier sensing functions: (i) physical carrier sensing and (ii) virtual carrier sensing. For physical carrier sensing traditional CSMA/CA is used. To determine whether the medium is available for transmission, before transmitting a new packet a station need to monitor the channel activity first. If the channel is idle for a period of time equal to the distributed inter-frame space (DIFS), the station starts to transmit instantly. Otherwise, if the channel is sensed busy, the station persists to monitor the channel until it is measured idle for a DIFS. At this point, it will wait for an additional DIFS interval and generate a random backoff timer before transmission in order to minimize the probability of multiple stations simultaneously starting transmission. Furthermore to avoid channel capture a station must wait a random backoff time between two consecutive packet transmissions, even if the medium is sensed idle for a DIFS period after the previous transmission.

For efficiency, DCF employs a discrete-time backoff scale scheme. The time right after an idle DIFS is slotted, and a station in only allowed to transmit at the beginning of each time slot.

Since the CSMA/CA can not rely on stations to detect a collision by listening to their own transmission, as it is done in IEEE 802.3 wired networks, an ACK is transmitted by the destination to signal the source of the successful packet reception. An ACK is transmitted after a short inter-frame space (SIFS) at the end of the received packet. This two-way handshaking technique for packet transmission is called basic access mechanism. 
Unlike the physical carrier sensing, for virtual carrier sensing DCF defines an optional four-way handshaking technique, called RTS/CTS handshake and NAV (network allocation vector) scheme is used as shown in Fig. 2. Here, if a node has a packet to send, which is larger than the RT, it first tries to reserve the channel by sending an RTS frame. Here, if a node has a packet to send, which is larger than the RT, it first tries to reserve the channel by sending an RTS frame by following the backoff procedure as the basic mechanism. After that, instead of sending the data frame, it sends a special short control frame called RTS. This frame includes the information about the source, destination and duration required by the following transaction - CTS, DATA and ACK transmission. Upon receiving the RTS, the destination node responds with another control frame called CTS, which also contains the same information. The transmitting station allowed to send data if the CTS frame is received correctly. All other nodes overhearing either RTS or CTS frame adjust their Network Allocation Vector (NAV) to the duration specified in RTS/CTS frame.

The NAV contains period of time in which the channel will be unavailable and is used as virtual Carrier sensing. Stations defer transmissions if either the physical or virtual Carrier sensing finds the channel being busy. Thus RTS/CTS mechanism has the ability of early detection of collision. But [16] suggested that RTS/CTS can also induce congestion, due to medium access control, which is different from the congestion that arises in the familiar TCP context.

RTS Threshold (RT) is a switch for the RTS/CTS mechanism. Packets that can be sent with a collision probability less than or equal to the collision probability of a RTS packet should be sent directly. Because RTS/CTS consumes extra bandwidth which has a negative effect on the performance of the network. The RTS/CTS mechanism is very effective in terms of system performance, especially when large packets are considered, as it reduces the length of the frames involved in the contention process.

\subsection{Related works}

In $[4,5]$ studies have been carried out on the performance evaluations of both the schemes mentioned in the previous section. The authors in [4,5] first conducted simulations to study the performance of RTS/CTS mechanism in IEEE 802.11 WLANs. In particular [6,8], pointed out that the RTS/CTS handshake does not work as well as it is expected in dealing with the hidden station problem and reducing interference, even though it was mainly employed for that purpose [9] also revealed other shortcomings of RTS/CTS handshake that did not exist in the basic scheme. Bianchi in [11] proved the superiority of RTS/CTS in highly loaded networks by calculating a theoretical upper limit for the throughput, based on a simplified chain model without taking into account packet retry limits. The authors in [12] have performed a simulation study, opted for maximum collision avoidance and suggested that the RTS/CTS mechanism must be employed at all times. On the other hand [13], illustrated that the RTS/CTS mechanism provides very limited advantages with respect to the basic access scheme, when no hidden stations are present, especially at high data rates (5 Mbps and $11 \mathrm{Mbps})$, knowing that the control packets (RTS, CTS and ACK) are always transmitted at either (1 Mbps) or (2 Mbps) [14,15] worked on developing an RT expression that relies on calculating the average overheads of both schemes assuming ideal conditions (ignoring hidden stations or transmission errors), which are redundant and unobtainable in the real world. Other work [17] involves optimization of RT based on a power management scheme to improve the average energy consumption by the packets. Effect of RTS threshold on network performance is observed by Sheu et al. [12]. Based on a mathematical model the average time of a successful and unsuccessful transmission is worked out in [10] and tried to establish a relationship between network saturation throughput and RTS threshold. The impact of using or disengaging the RTS/CTS mechanism in IEEE 802.11 DCF MAC protocol is also observed in [7]. These works show the interest and importance of RTS threshold (RT) and how an appropriate value may extract efficient transmission. 


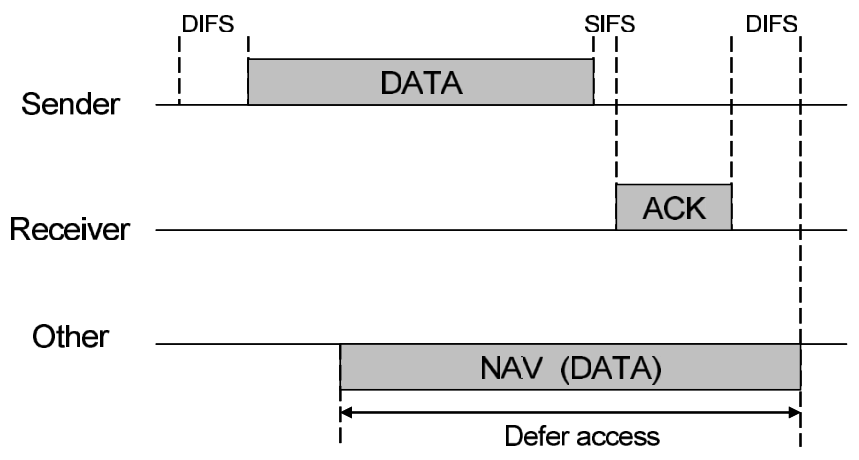

Fig. 1. Basic CSMA/CA access mechanism.

\section{Numerical comparison}

The maximum throughput for an ad hoc network in saturation mode is calculated as in [11] and it is as follows:

$$
S=\frac{P_{t r} P_{s} \text { Data }}{\left(1-P_{t r}\right) \sigma+P_{t r} P_{s} P_{s}+P_{t r}\left(1-P_{s}\right) T_{c}}
$$

Where, $P_{t r}$ is the probability of at least one transmission, $P_{s}$ is the conditional probability of success of an occurring transmission, $T_{s}$ is the average duration of a successful transmission, $T_{c}$ is the average duration of a collision, $\sigma$ is the duration of a single slot time. $P_{t r}$ and $P_{s}$ are functions of the network size, $n$ is the probability of a packet transmission at slot time $\tau$, which depends only on the initial backoff window size and its stage limit $m$.

$$
P_{t r}=1-(1-\tau)^{n}, P_{s}=n \tau(1-\tau)^{n-1} / P_{t r}
$$

Where,

$$
\begin{array}{r}
\tau=\frac{2(1-2 p)}{(1-2 p)(W+1)+p W\left(1-(2 p)^{m}\right)} \\
p=1-(1-\tau)^{n-1}
\end{array}
$$

$T_{s}$ and $T_{c}$ depend on the transmission scheme.

From Fig. 1 we have:

$$
\left\{\begin{array}{l}
T_{s}^{\text {Basic }}=D I F S+T_{\text {header }}+\frac{\text { Data }}{R}+S I F S+T_{A C K}+2 \delta \\
T_{c}^{\text {Basic }}=D I F S+T_{\text {header }}+\frac{D a t a}{R}+\delta
\end{array}\right.
$$

And from Fig. 2:

$$
\left\{\begin{aligned}
& T_{s}^{R T S}=D I F S+ 3 S I F S+T_{R T S}+T_{C T S}+T_{\text {header }}+ \\
& \frac{\text { Data }}{R}+S I F S+T_{A C K}+4 \delta \\
& T_{c}^{\text {Basic }}=D I F S+T_{R T S}+\delta
\end{aligned}\right.
$$

Here $\delta$ is the propagation delay and $\mathbf{R}$ is the data rate. 


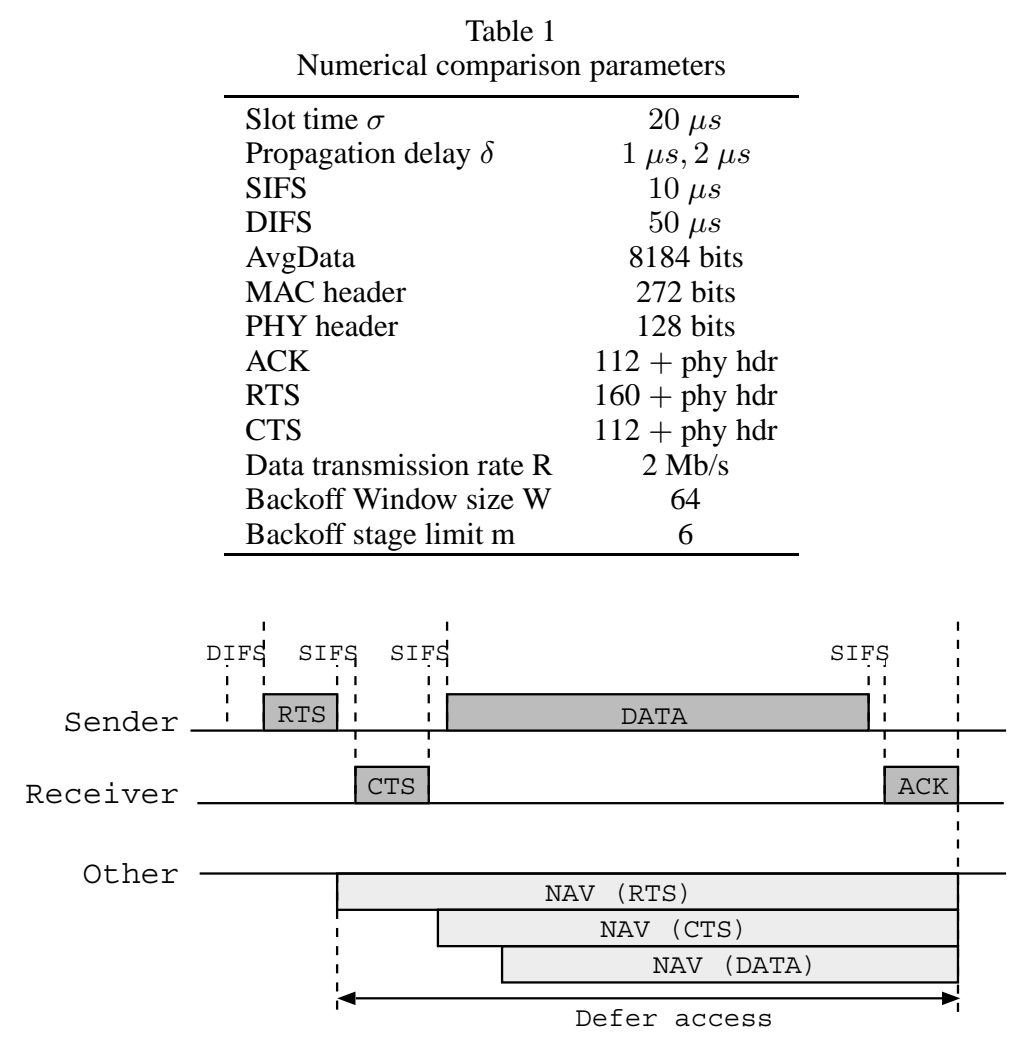

Fig. 2. RTS/CTS mechanism of DCF.

A numerical comparison between the maximum throughput of each scheme can be obtained by substituting in expression (1) with the parameters shown in Table 1.

As shown in Fig. 3, extra overhead introduced by RTS and CTS packets causes the handshake scheme to have lower throughput than the basic scheme in case of small network size, but performs much better in case of large number of contending nodes.

Node density and traffic can be constantly changing in mobile ad hoc networks. Consequently, nodes have to adapt their transmission schemes according to those changes, and it is imperative to find a way to dynamically switch between the two schemes and adjust RT irrespective of the number of surrounding nodes. In the following section we will introduce our dynamically adjusting scheme for adaptive transmission control.

\section{Proposal}

\subsection{Adaptive transmission control scheme}

If there is a small number of contending nodes and a low collision risk, in other words the packet delivery ratio is high, then the packets should be sent directly using the basic scheme. If that ratio drops below a certain threshold, RTS/CTS handshaking should be used. Hence, periodical adjustment of RT to the packet delivery ratio is necessary for optimal throughput. 


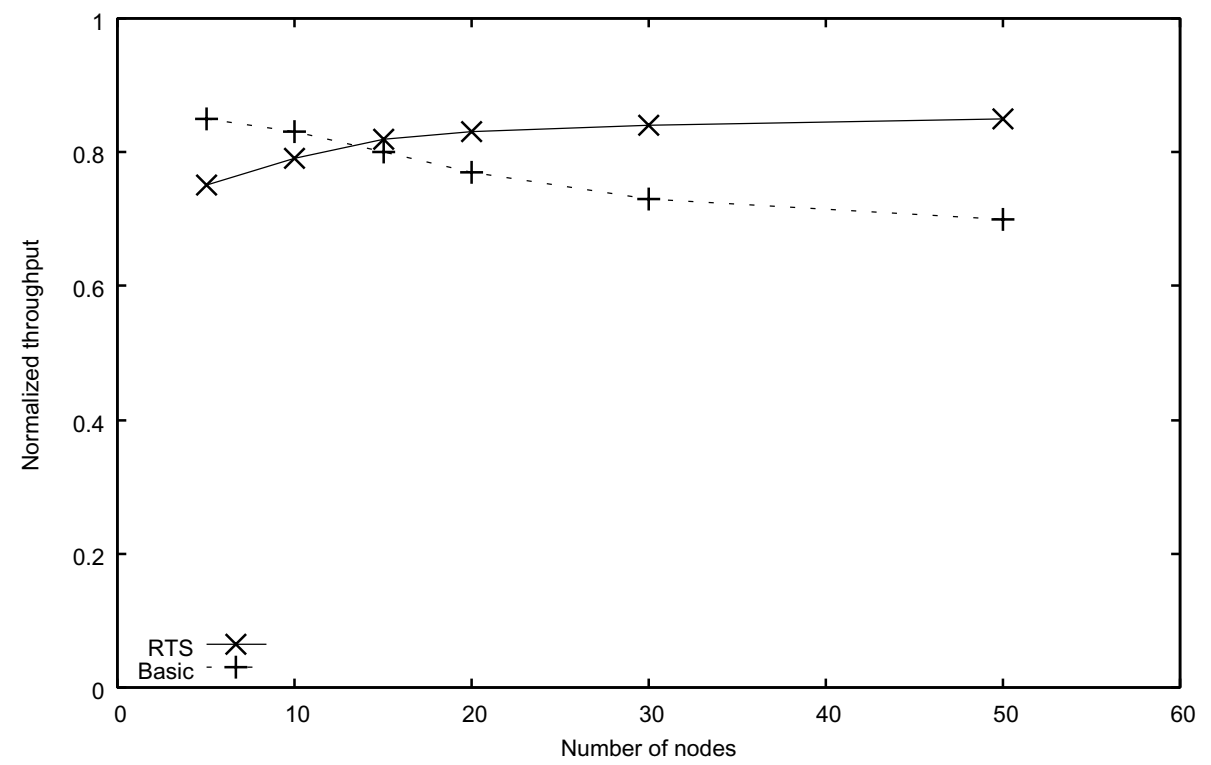

Fig. 3. Numerical comparison of maximum throughput between CSMA and RTS/CTS.

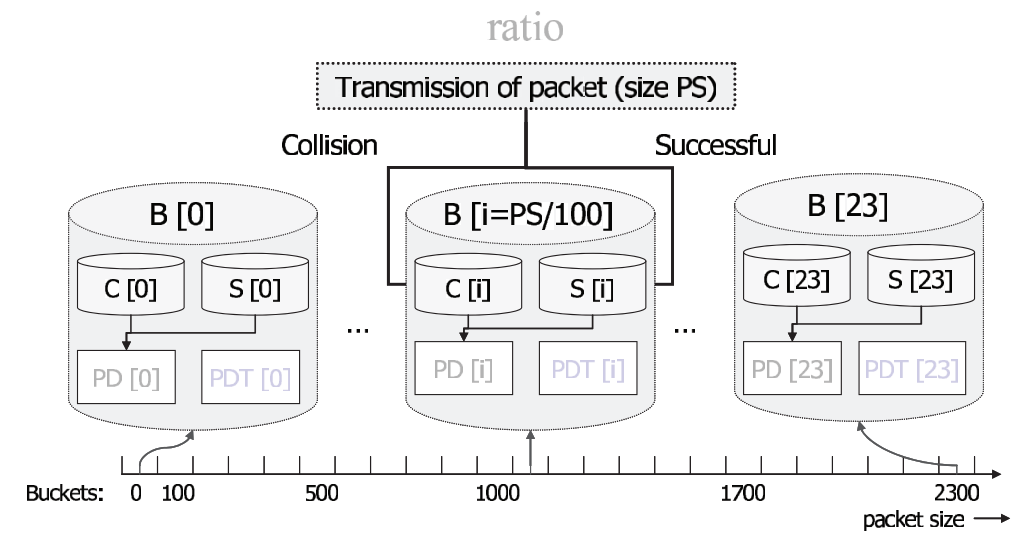

Fig. 4. Short term storage of success ratio.

Collision probability increases with the increase in the size of data packets and as a consequence the packet delivery ratio drops. Therefore, this threshold has to be accordant with the packet size. Each bucket $(B[i])$ contains the successful $(S[i])$ and unsuccessful $(C[i])$ packet index and the indexing is done by $B[i=P S / 100]$, as shown in Fig. 4. The maximum packet size used for transmission in 802.11 is 2400 bytes. Now, divide that $[0,2400]$ scale into 24 parts (or equal segments) and call each of them buckets.

- Number of successfully transmitted packets $S[i]$.

- Number of collisions $C[i]$.

- Packet delivery ratio $P D[i]=\frac{S[i]}{S[i]+C[i]}$.

- Constant packet delivery ratio threshold $P D T[i]$ to determine whether $P D[i]$ is satisfactory or not.

PDT characteristic is obtained through simulation determining a satisfactory delivery ratio for each 


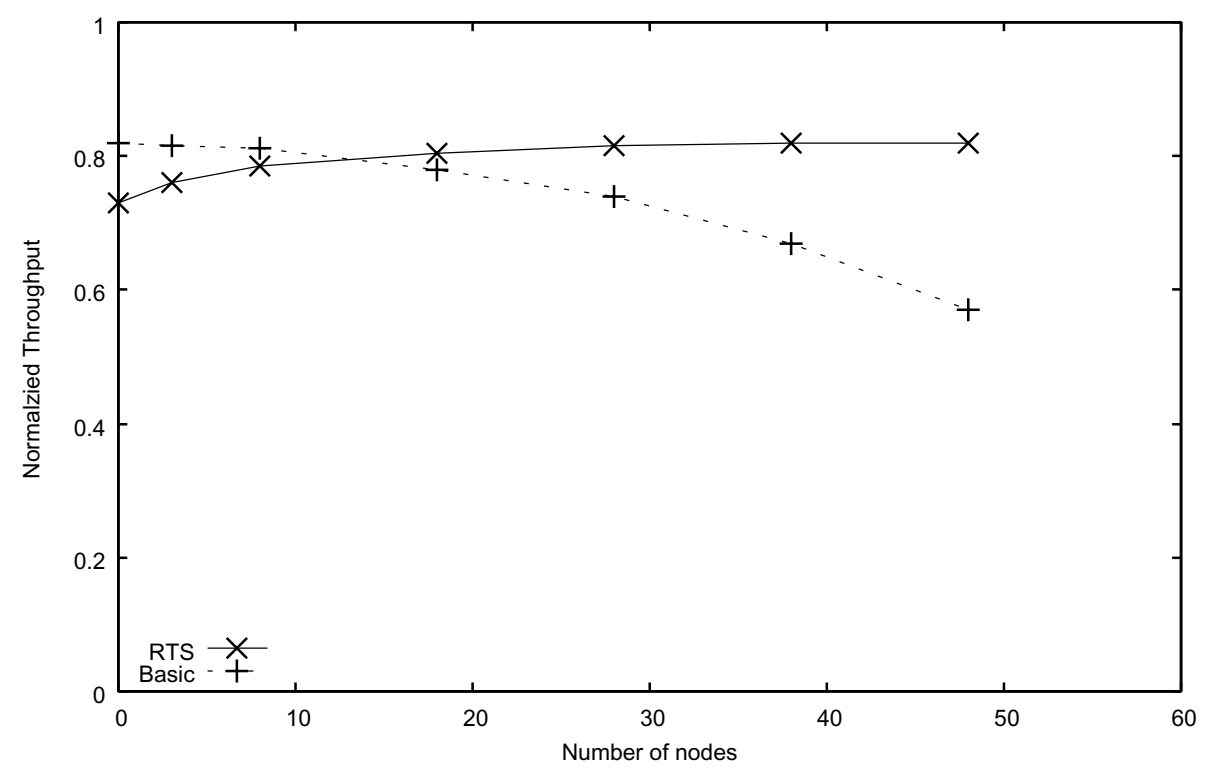

Fig. 5. Throughput between node A and B (packet size $=1000$, data rate $=2 \mathrm{Mbps}$ ).

bucket using the basic scheme.

\subsection{Investigation of PDT}

Most widely recognized network simulator, ns- 2 is used to evaluate the effectiveness of transmission schemes. The network model is a multi-hop wireless topology using AODV [18] as routing protocol. The link layer is a shared media radio with nominal channel bit rates of 2, 5 and $11 \mathrm{Mbps}$. We run the simulation on the $1000 \times 1000 \mathrm{~m}^{2}$ field for 300 seconds. Nodes are moving according to the Random Waypoint model [19], with parameters: maximum speed $=20 \mathrm{~m} / \mathrm{sec}$, minimum speed $=0$, pause time $=30$ secs. Every plots in these graphs is the average of at least 10 simulations. There are 24 pairs of source-destination exchanging random size CBR packet between themselves, which is considered as background traffic. Only one static pair A and B are exchanging constant size packet and they are in one hop distance, which is the observed pair in our experiment. One hop distance is considered as it produces maximum packet delivery, which we required for determining the packet deliver thresholds.

For both schemes, we calculate for each bucket the throughput and the end-to-end delivery ratio between two static nodes A and B, as the offered load gradually increases. The offered load is varying with the number of pairs increasing from 0 to 25 or number of nodes increasing from 0 to 50 . For instance, the results for bucket $\mathrm{B}[10]$ (packet size $=1000$, data rate $=2 \mathrm{Mbps}$ ) are presented in Figs 5 and 6.

When offered load is low, RTS/CTS handshaking has lower throughput due to extra overhead. On the other, when the offered loads is high, throughput of the basic drops because of high collision probability. Both schemes have equal throughput when number of pairs is less than 6 , where the delivery ratio for the basic scheme is $95 \%$. Given this, if the delivery ratio is kept above $95 \%$, highest possible throughput can be maintained at any offered load. That means, when transmitting packets with size only between 1000 and 1100 , using basic scheme will assure a satisfactory packet delivery with a higher throughput. As the offered load increases, the delivery ratio is expected to drop. When it reaches below 95\%, switching 


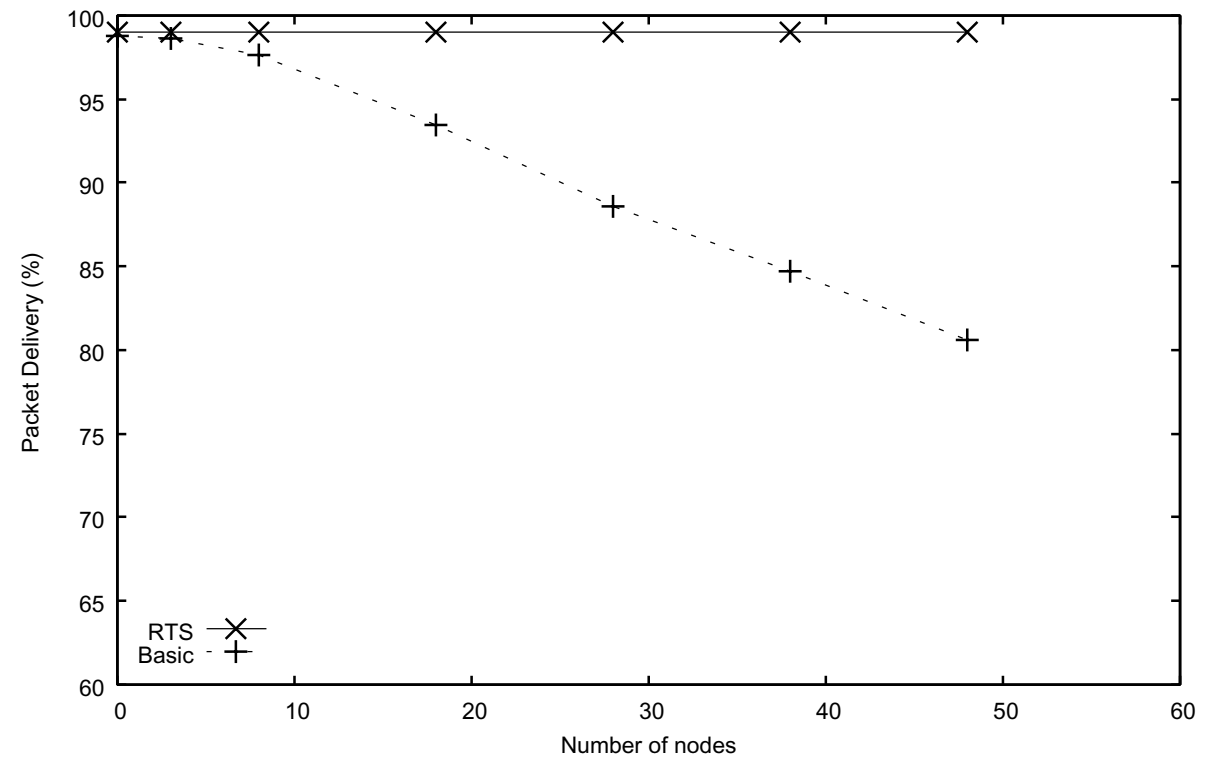

Fig. 6. Packet delivery ratio between A and B (packet size $=1000$, data rate $=2$ Mbps).

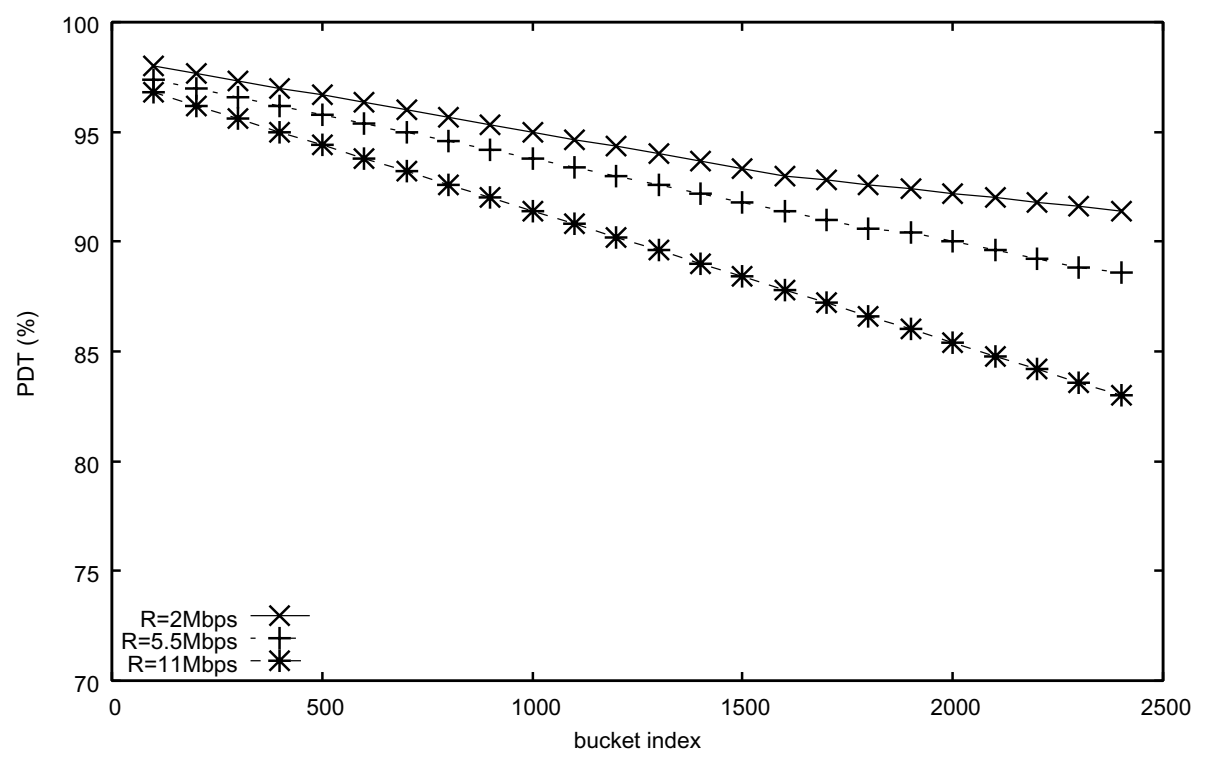

Fig. 7. Characteristics of packet delivery ratio threshold.

to RTS/CTS will maintain the high throughput. Thus, by gathering these optimal packet delivery ratios corresponding to each bucket, we can obtain for each nominal channel bit rate. The packet delivery thresholds are shown in Fig. 7.

Based on this packet delivery threshold characteristics, the proposed algorithm will store success history of transmissions of each bucket, calculate their delivery ratios while ignoring those with low number of transmissions, compare them to their delivery ratio thresholds respectively, and adjust RT accordingly after every equal time interval. The RT updating of interval is inversely relative to the data 


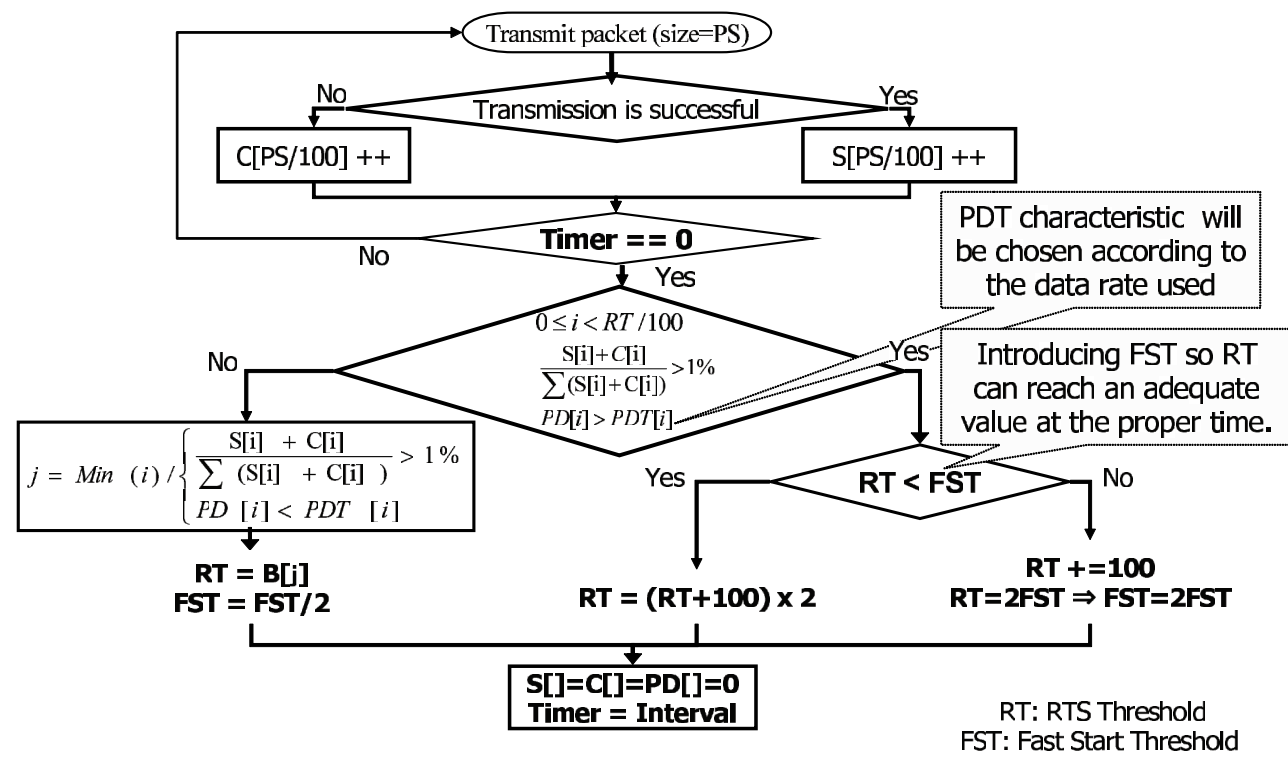

Fig. 8. Algorithm flowchart.

sending rate to make sure there will be enough history of transmissions to operate on.

In the real world, RT is set to a MTU $=2400$ bytes and RTS/CTS is never used assuming low node density and traffic as the likely circumstances. Considering this as the initial value for RT and after a transmission course, if all bucket have satisfactory delivery ratio, RT will be increased. Otherwise, RT will be set to the lowest bucket with unsatisfactory delivery ratio. As shown in the flow chart of the algorithm (Fig. 8), the amount by which RT changes is controlled by a Fast Start Threshold (FST) parameter to help it reaches an adequate value at the proper time. After RT is adjusted the counters are reset and the timer is set back to the interval value.

\section{Evaluation}

\subsection{Analytical evaluation}

For analytical evaluation, the distribution function of node density along the network has to be defined in order to be able to see how that affects the performance of the Adaptive Scheme which is based on adapting the transmission of mobile nodes to their surrounding environment. That also will help us to predict how node density is going to affect the probabilities of Basic and RTS/CTS schemes usage along the network.

Here, node density $\mu$ for a node at point $(x, y)$ is defined as the number of existing node in 1-hop distance in the network.

Assuming there is no idle nodes and all nodes are trying to transmit, no collision will occur if:

$$
\forall(x, y) 0 \leqslant \mu(x, y)<\frac{2}{\text { networksize }} .
$$

As the node density gets higher, there is more chance of packet collision and therefore Packet Delivery (PD) drops. 


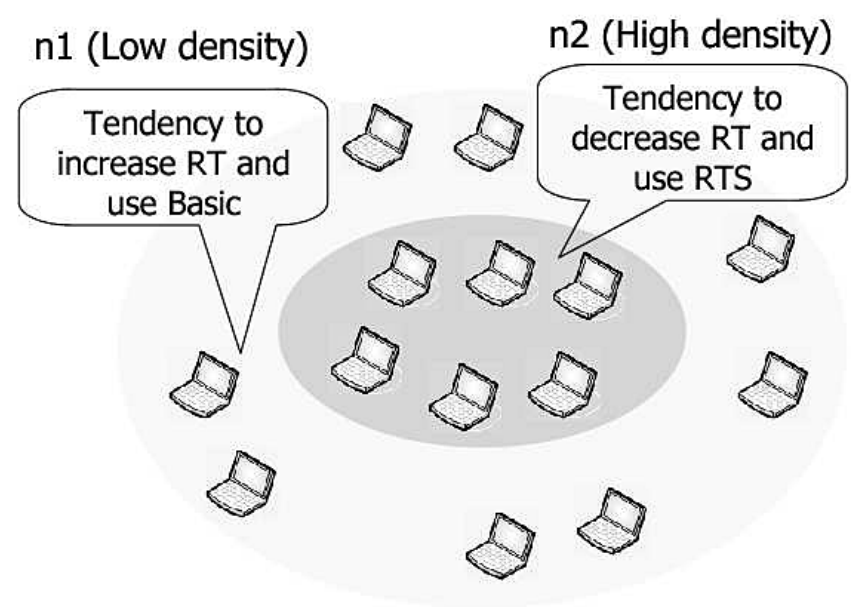

Fig. 9. Network model: n1 with low traffic and n2 with high traffic load.

Now, that the node density is defined, it is possible to calculate the throughput of the Adaptive Scheme used in a certain network model. Since, the adaptive scheme's main function is to adapt the RTS Threshold (RT) to the changes in the network topology and provide both collision avoidance in dense parts of the network and maximize the channel utilization in the sparse parts, it is more logical to evaluate the algorithm in a network model where the variety in node density is visible. We consider a network model as illustrated in Fig. 9. It consists of two areas, with varying node density. Node density is high in the center of the area and sparse around the outer area, which is more or less like a realistic scenario of an urban area. The Adaptive algorithm will perform in such a way that nodes in the sparse area will have tendency to maximize the channel utilization since collisions are unlikely to occur and therefore the adaptive scheme will gradually increase the RTS Threshold (RT) in order to use the Basic scheme. Similarly, as transmitting nodes get closer to the center, they will have to avoid collisions by lowering their RT and use the RTS/CTS scheme.

Let $P_{\text {Basic }}=1-P_{\mathrm{RTS}}$ be the probability of using the Basic scheme. According to the definition of node density distribution, these probabilities in the network under consideration are:

$$
P_{\text {Basic }}=P\left(0 \leqslant \mu(x, y)<\frac{2}{\text { networksize }}\right)=\sum \frac{1}{n_{1}+n_{2}} \cong \frac{n_{1}}{n_{1}+n_{2}}
$$

The probability of using the basic scheme within this network model is equal to the probability of nodes having at most one neighboring node with 1-hop distance. In general, the probability is the number of nodes in the sparse mode area over the total network size.

$$
P_{\mathrm{RTS}}=P\left(\frac{2}{\text { networksize }} \leqslant \mu(x, y)\right) \cong \frac{n_{2}}{n_{1}+n_{2}}
$$

Contrary to the Basic scheme, the probability of using the RTS/CTS scheme within the network model is same as that of nodes having at least one neighboring node within 1-hop distance. That means, the probability of using RTS/CTS is the number of nodes in the dense mode area over the total network size.

The throughput of the adaptive scheme is

$$
S_{\text {Adap }}=\frac{P_{t r} P_{s} \text { AvgData }}{\left(1-P_{t r}\right) \sigma+P_{t r} P_{s} T_{s}^{\text {Adap }}+P_{t r}\left(1-P_{s}\right) T_{c}{ }^{\text {Adap }}}
$$


Where,

$$
\left\{\begin{array}{l}
T_{s}{ }^{\text {Adap }}=P_{\text {Basic }} T_{s}{ }^{\text {Basic }}+P_{R T S} T_{s}{ }^{R T S} \\
T_{c}{ }^{\text {Adap }}=P_{\text {Basic }}^{2} T_{c}{ }^{\text {Basic } / \text { Basic }}+ \\
\quad 2 P_{\text {Basic }} P_{R T S} T_{c}^{\text {Basic } / R T S}+P_{R T S}^{2} T_{c}{ }^{R T S / R T S}
\end{array}\right.
$$

Here, $P_{R T S}$ and $P_{\text {Basic }}$ is the probabilities of using RTS/CTS and Basic schemes respectively, and are calculated previously as functions of the density sparsity parameters $n_{1}$ and $n_{2}$ in Eqs (5) and (6).

Successful transmission time for the Adaptive scheme is depending on which area the transmission took place, combining the duration times of a successful transmission using Basic scheme or RTS scheme. On the other hand, collision time for the Adaptive scheme is combined from the following 3 cases:

1. Collision of two control frames RTS $T_{c}{ }^{R T S / R T S}$

2. Collision of a Basic data frame and a control frame RTS $T_{c}{ }^{\text {Basic/RTS }}$

3. Collision of two Basic data frames $T_{c}$ Basic/Basic

With the first case throughput of RTS/CTS scheme can be calculated. The second case involves the data size distribution $F(D a t a)$ as the overlapping time between a control frame, and control frame depends on the size of the latter. For the third case, the collision of two data frames is null since it would occur only in the area where the basic scheme is used, which is assumed as collision free according to the network model.

$$
\left\{\begin{aligned}
T_{c}^{R T S / R T S}= & R T S+D I F S+\delta \\
T_{c}^{\text {Basic } / R T S}= & T_{\text {header }}+\text { DIFS }+\delta+ \\
& 1 / R \int_{0}^{\text {AvgData }} 1-F(\text { Data }) d \text { Data } \\
T_{c}^{\text {Basic/Basic }}= & 0
\end{aligned}\right.
$$

For the second case, a random uniform distribution would generate a collision time equal to that of generated by the basic scheme.

$$
1 / R \int_{0}^{\text {AvgData }} 1-F(\text { Data }) d \text { Data }=\frac{\text { AvgData }}{R}
$$

A numerical comparison between the three schemes can be obtained by substituting the parameters given in Table 1.

The network density is chosen as $n_{2}=4 n_{1}$. As shown in Fig. 10, extra overhead introduced by RTS and CTS packets causes the handshake scheme to have lower throughput than the basic scheme in case of low network size, but performs much better in case of large number of contending nodes. On the other hand, the Adaptive scheme outperforms both traditional scheme by providing maximum channel utilization for the nodes that were using RTS/CTS when there is no need for that while ensuring high collision penalty as they roam between dense and sparse area. Also as control frames, RTS, CTS and ACK are always sent at $2 \mathrm{Mbps}$, large data packets can be sent without channel reservation since they appear to be relatively smaller at higher data rates and the overlapping time that leads to collisions becomes smaller too. As a result Adaptive scheme shows better performance where data rate is considerably high (5 Mbps, $11 \mathrm{Mbps}$ ) compared to control frames rate (2 Mbps).

Performance of the Adaptive scheme changes as the network density distribution varies. In a scenario where $n_{2}=0$, i.e. network consists of only sparse node, Adaptive scheme appears as a dominant Basic scheme with no occurrence of collision, and network throughput is at its highest as shown in Fig. 11. 


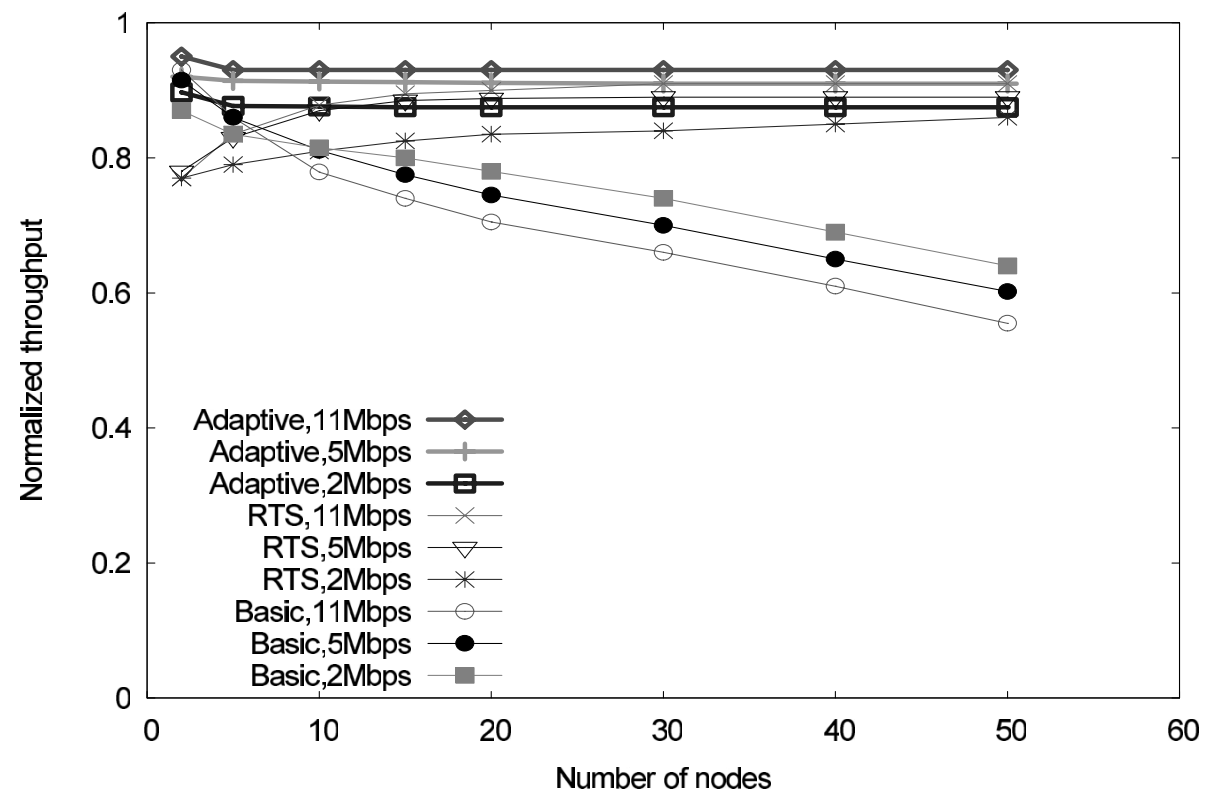

Fig. 10. Numerical throughput of the Adaptive scheme, Basic CSMA/CA and RTS/CTS.

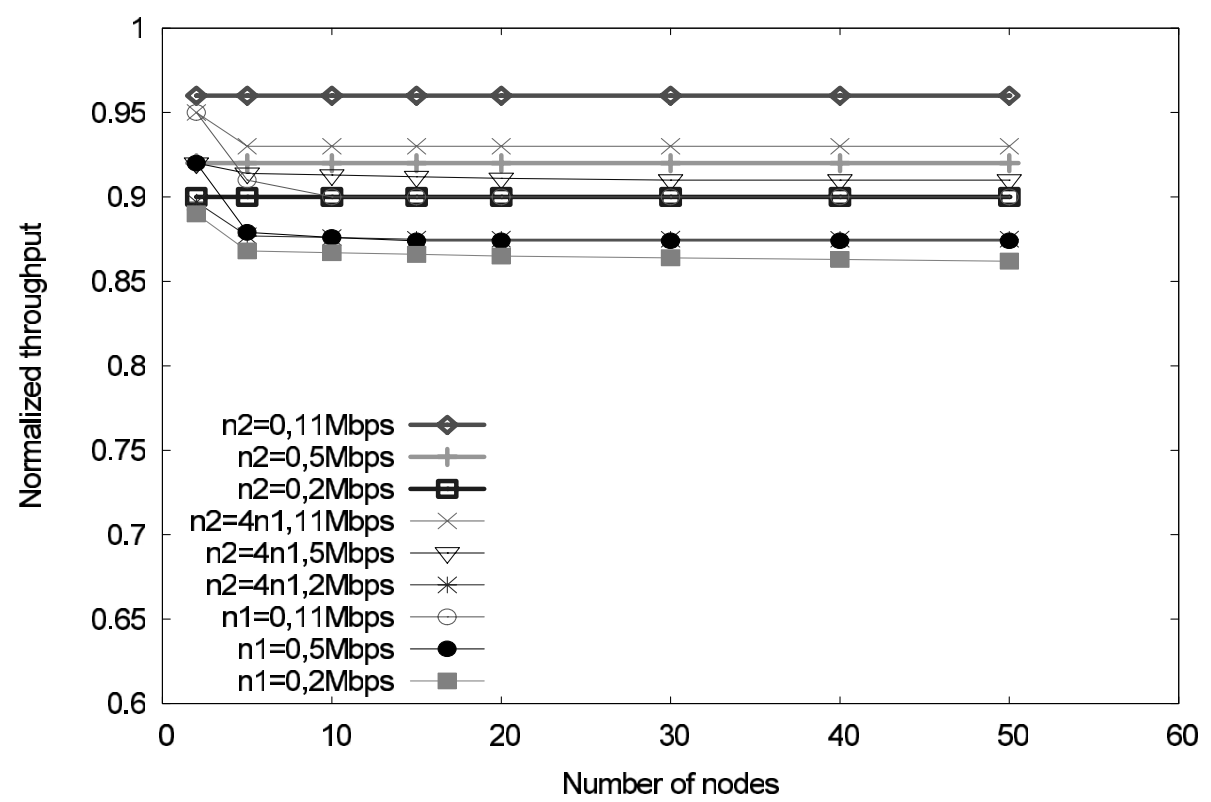

Fig. 11. Numerical throughput of the Adaptive scheme with varying node density distribution.

But when $n_{1}=0$, i.e. network consists of only densely populated nodes, the dominant scheme is RTS/CTS and our proposed Adaptive scheme will still maintain a high throughput under the constrained circumstances. 


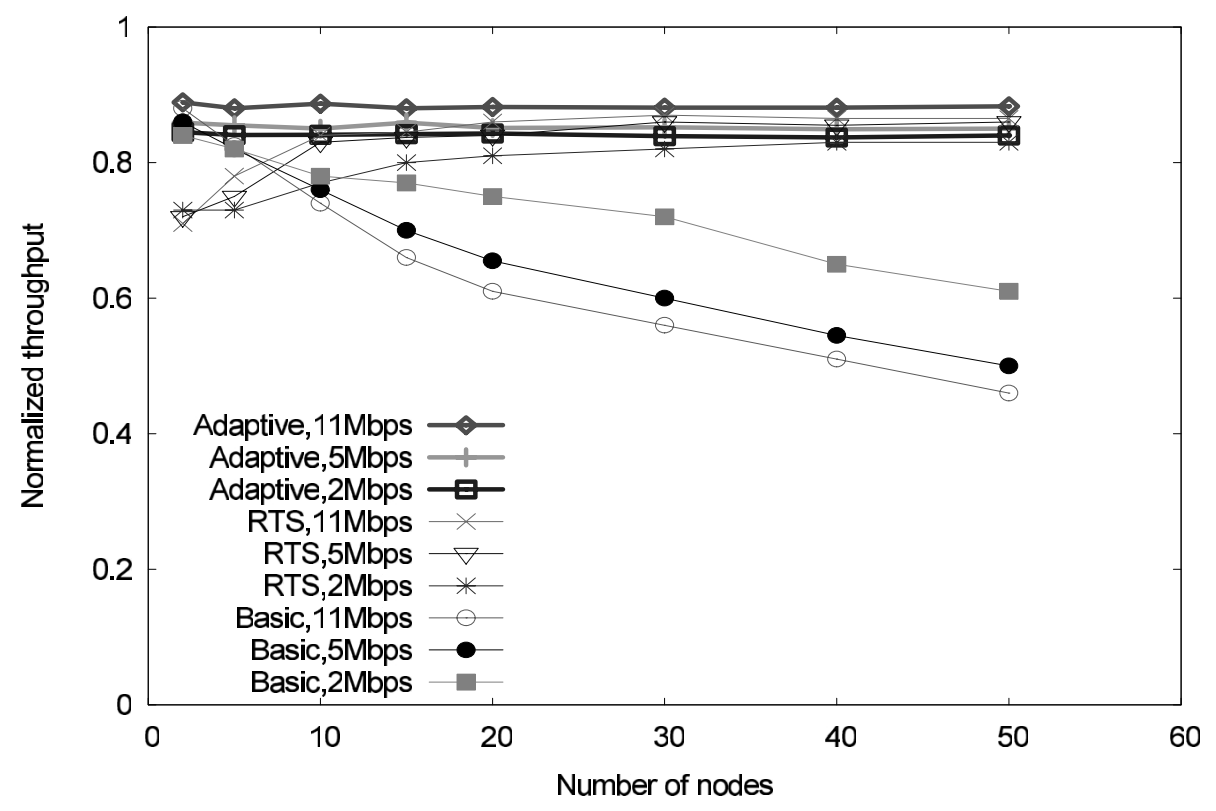

Fig. 12. Comparison of Throughput between Adaptive, CSMA and RTS schemes.

\subsection{Simulation based Evaluation}

Adopting the same network topology used for the numerical evaluation, the network model is combined of two areas of different node densities, as illustrated in Fig. 9. We evaluated the proposed algorithm and compared it to the other schemes through simulation done in ns-2. Network traffic parameters are similar to the simulation environment used for the investigation of PDT. Data size exchanged between the nodes is random and the performance metric in our observation is the throughput of the whole network.

As expected from the analytical evaluation, the graphs in Figs 12 and 13 show that our proposed scheme outperforms the basic and the RTS schemes. After the first Interval of time, nodes in different areas ( $n 1$ and $n 2)$ using our algorithm were able to calculate the success ratios and adjust their RTS Thresholds (RT) to an adequate value to sustain a good packet delivery ratio as well as to maintain the throughput to the highest possible level. The performance of the algorithm improves with time as we are accumulating the information regarding the success ratio and can more correctly calculate the threshold value. The performance improves as the data rate increases compare to control frame rate. Also, as sparse the nodes in the network, more bandwidth can be utilized by omitting RTS/CTS handshaking and its related extra transmission.

Besides that, as control frames, RTS, CTS and ACK, are always sent at $2 \mathrm{Mbps}$, large data packets can be sent without channel reservation since they appear to be relatively smaller at higher data rates. As a result Adaptive scheme shows better performance as shown in Fig. 12 where the data rate $(5,11 \mathrm{Mbps})$ is considerably high compared to control frames rate $(2 \mathrm{Mbps})$. Figure 13 illustrated the throughput of Adaptive scheme with varying node density.

Comparison between analytical evaluation and simulation based evaluation results are shown in Figs 14 and 15. The analytical evaluation does not take into account the parameters such as routing related traffic which is considered in network simulation. Hence, higher throughput is obtained in analytical evaluation.

The proposed scheme provides better channel utilization in the low traffic area $(n 1)$ and higher collision penalty in the area with high traffic $(n 2)$. 


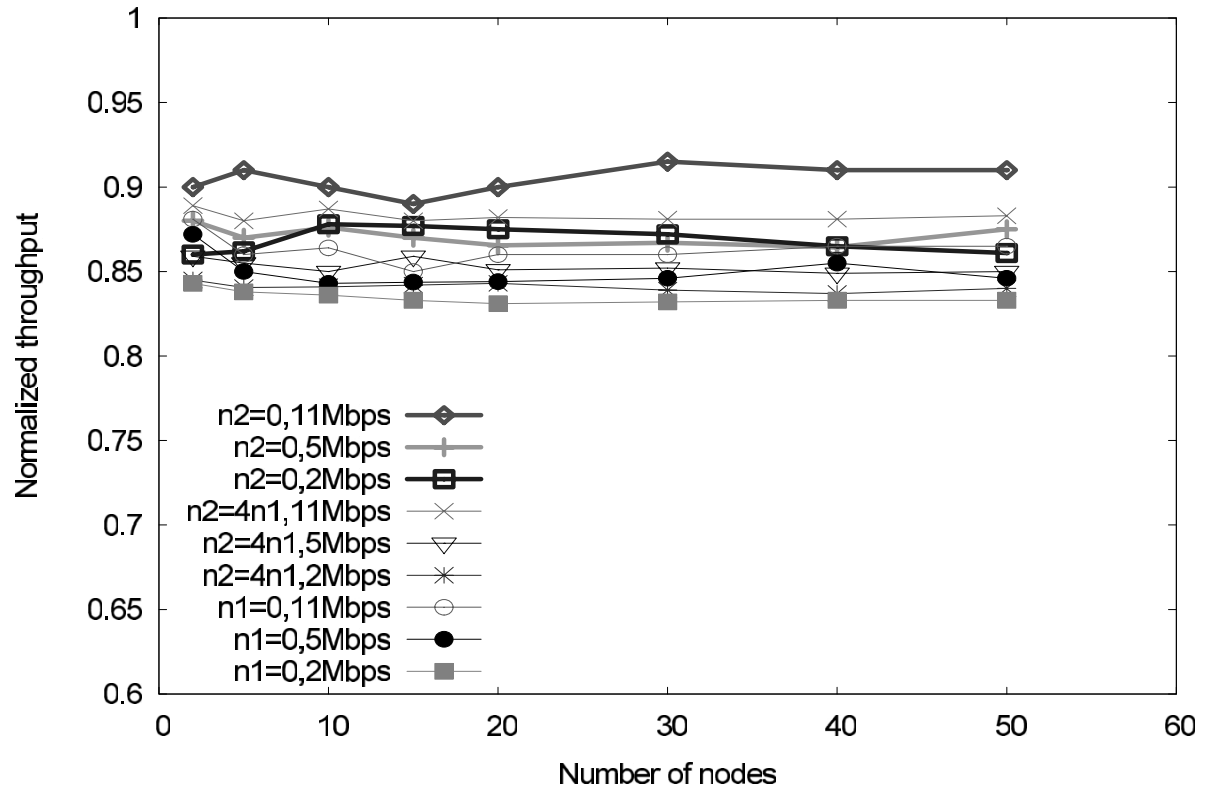

Fig. 13. Throughput of the Adaptive scheme with node density distribution.

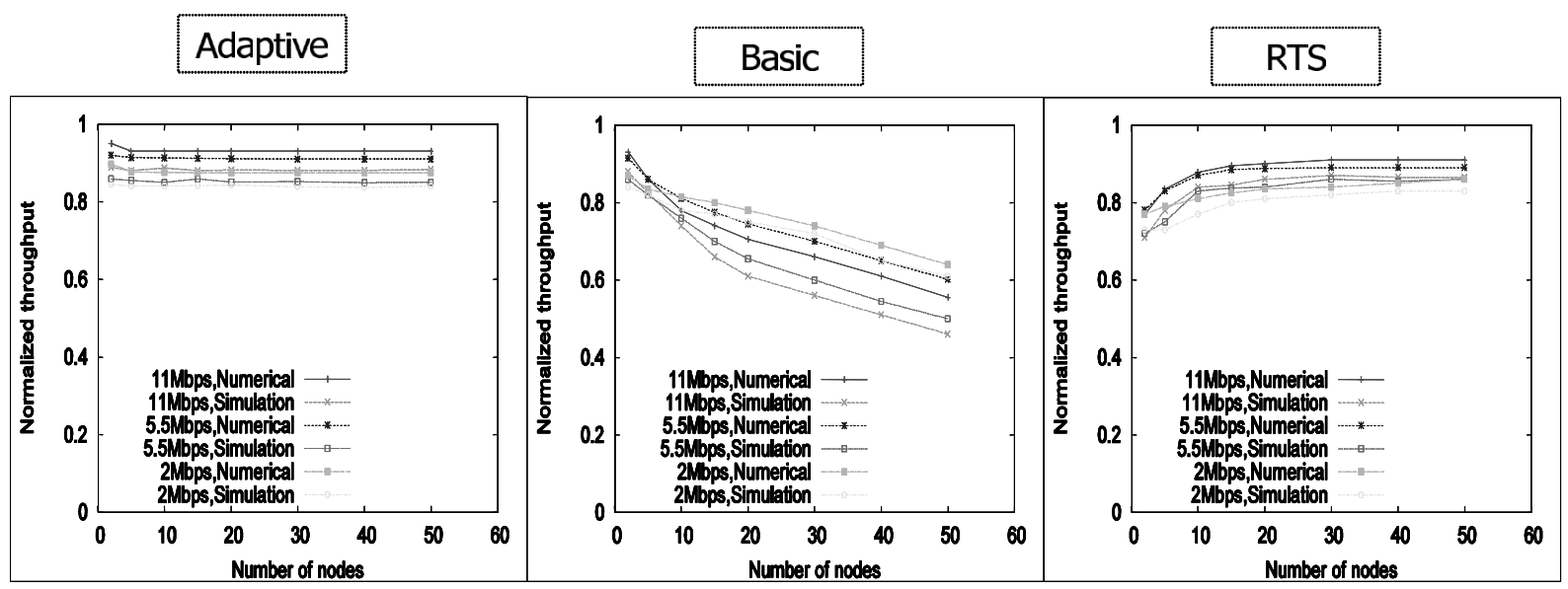

Fig. 14. Throughput of the Adaptive, Basic and RTS/CTS scheme when $n_{2}=4 n_{1}$.

\section{Conclusion}

In this paper we have shown how the performance of basic CSMA/CA and the optional RTS/CTS fourway handshaking mechanism employed by IEEE 802.11 MAC protocol depend on network topology and traffic load. We have also shown the significance of RTS Threshold (RT), a key to the switching between the two schemes can affect the performance of transmission between nodes. As the network topology, node density and traffic is constantly changing, we argued that efficient handling of RTS Threshold (RT) is imperative for better throughput.

In correspond to that we proposed a dynamic way to adjust the RT according to the packet delivery ratio, which is an indicator of the network situation. We demonstrated through both analytical and 


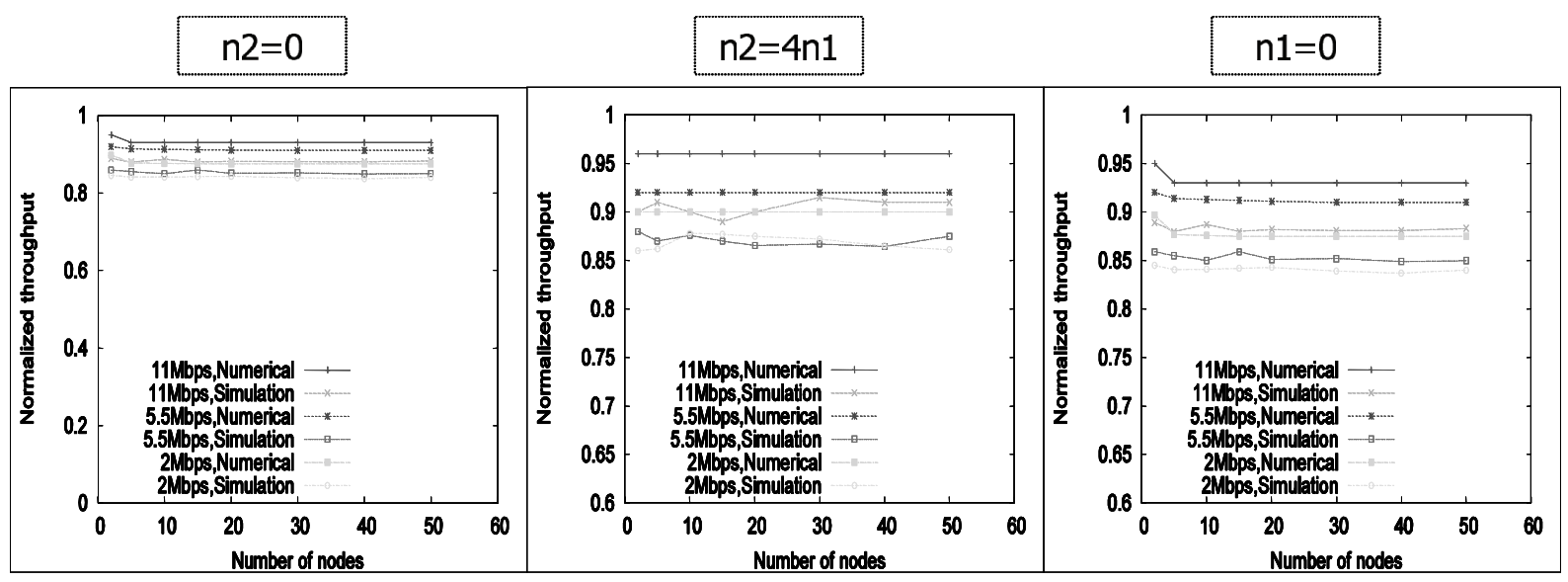

Fig. 15. Throughput of the Adaptive scheme with varying node density distribution.

simulation evaluation that, how the proposed algorithm tunes up the transmission to an adequate value at a proper time irrespective of the number of contending nodes.

Evaluation results showed that the proposed adaptive transmission control scheme out-performs the basic and the RTS/CTS handshaking scheme. In this work, we have used an ns-2 based realistic model to evaluate our proposed scheme. If the network mobility and traffic is static, then the effectiveness our algorithm is nominal compared to the existing schemes. But in real-world, network traffic is constantly changing as various kinds of applications are running and as a result the range of packet size may also widely vary, and in such situation the effectiveness of our schemes will be more visible.

We further intend to compare our algorithm with other works related to dynamic change of RTS threshold and evaluate them on MobiREAL simulator [20], which is a realistic network simulator for MANET. MobiREAL provides a new methodology to model and simulate realistic mobility of nodes and enables to evaluate MANET applications in more actual environments.

\section{Acknowledgment}

This work was partially supported by Ministry of Internal Affairs and Communications in Japan, SCOPE project (071502003) and the Ministry of Education, Culture, Sports, Science and Technology, Grants-in-Aid for Scientific Research, 19200005, and Grant-in-Aid for Young Scientists (B), 20700056.

\section{References}

[1] IEEE 802.11 Working Group, Part 11: Wireless LAN Medium Access Control (MAC) and Physical Layer (PHY) Specifications, ANSI/IEEE Std. 802.11, Sept.1999.

[2] B.P. Crow, I. Widjaja, L.G. Kim and P.T. Sakai, IEEE 802.11 Wireless Local Area Networks, IEEE Communication Magazine 35(9) (Sept 1997).

[3] Wireless LAN Medium Access Control (MAC) and Physical Layer (PHY) Specification: High-Speed Physical Layer Extension in the $2.4 \mathrm{GHz}$ Band, IEEE 802.11b WG, 1999.

[4] J. Weinmiller, H. Woesner and A. Wolisz, "Analyzing and improving the IEEE 802.11-MAC protocol for wireless LANs", in Proc. of MASCOT, pp. 200-206, Feb.1996.

[5] J. Weinmiller, H. Woesner, JP Ebert and A. Wolisz, Analyzing the RTS/CTS mechanism in the DFWMAC Media Access Protocol for Wireless LANs, in Proc. Of PWC, pp. 117-130, April 1995. 
[6] K. Xu, M. Gerla and S. Bae, How Effective is the IEEE 802.11 RTS/CTS Handshake in Ad Hoc Networks?. Proc. of IEEE Globecom 1 (Taipei, 2002), 72-76.

[7] H.-J. Ju, I. Rubin and Y.-C. Kuan, An Adaptive RTS/CTS Control Mechanism for 802.11 MAC Protocol, IEEE VTC 2003-spring (April 22-2, 2003).

[8] K. Xu, M. Gerla and S. Bae, Effectiveness of RTS/CTS Handshake in IEEE 802.11 Based Ad Hoc Networks, Ad Hoc Networks Journal 1(1) (July 2003), 107-123.

[9] J.L. Sobrinho, R. de Haan and J.M. Brazio, Why RTS-CTS is not your ideal wireless LAN multiple access protocol, WCNC05, New Orleans, Mar. 2005.

[10] S. Yan, Y. Zhuo, S. Wu and W. Guo, Adaptive RTS threshold for maximum network throughput in IEEE 802.11 DCF, Wireless communications and networks, Wuhan, 4-6 November 2003.

[11] G. Bianchi, Performance Analysis of the IEEE 802.11 Distributed Coordination Function, IEEE Journal on Selected Area in Comm 18(3) (Mar 2000), 535-547.

[12] S.-T. Sheu, T.Chen, J.Chen and F. Ye, The impact of RTS Threshold on IEEE 802.11 MAC protocol, in Proc. of IEEE ICPADS, pp. 267-272, Dec. 2002.

[13] R. Bruno, M. Conti and E. Gregori, IEEE 802.11 optimal performance: RTS/CTS mechanism vs. basic access, Proc. of IEEE PIMRC 4 (Sept. 2002), 1747-1751.

[14] P. Chatzimisios, A.C. Boucouvalas and V. Vitsas, Optimization of RTS/CTS handshake in IEEE 802.11 wireless LANs for maximum performance, Global Telecommunication Conference Workshops, 2004. IEEE 29 Nov.-3 Dec. 2004. Page(s) 270-275

[15] L. Zhang and Y. Shu, RTS threshold self-tuning algorithm based on delay analysis on 802.11 DCF, Wireless Communications, Networking and Mobile Computing (Sept. 2005) 627-630.

[16] S. Ray, J. Carruthers and D. Starobinski, RTS/CTS-induced congestion in ad-hoc wireless LANs, in Proceedings of IEEE WCNC, pp. 1516-1521, March 2003.

[17] S. Yan, Y. Zhuo and S. Wu, An adaptive RTS threshold adjust algorithm based on minimum energy consumption in IEEE 802.11 DCF, Communication Technology Proceedings, 2003. ICCT 2003. International Conference on Volume 2, 9-11 April 2003 Page(s) 1210-1214 vol.2.

[18] C.E. Perkins and E.M. Royer, Ad Hoc On-demand Distance Vector Routing, Proc. the 2nd IEEE WMCSA'99, New Orleans, LA, February 1999, pp. 90.100.

[19] T. Camp, J. Boleng and V. Davies, A Survey of Mobility Models for Ad Hoc Network Research, Wireless Comm. \& Mobile Computing (WCMC): Special issue on Mobile Ad Hoc Networking: Research, Trends and Applications 2(5) (August 2002), 483-502.

[20] Designed by MobiREAL Team in Mobile Computing Lab.(Higashino Lab.), Graduate School of Information Science and Technology, Osaka University, Japan http://www-higashi.ist.osaka-u.ac.jp/.

Mostafa Mjidi received his M.S. degree in Information Science from Graduate School of Information Science, Tohoku University, Japan, in 2008,. He is the recipient of Student encouragement award from IEEE Tohoku Section, Japan, in 2006. Currently he is working in NTT Communication Corp. Global IP Network division.

Debasish Chakraborty received his doctoral degree from the Graduate School of Information Science, Tohoku University, Japan, in 1999. Presently he is working as a visiting Associate Professor in the Research Institute of Electrical Communication, Tohoku University. He was a TAO research fellow and a NiCT foreign research fellow at Tohoku University Research Center. His main research interests are multicast routing algorithm, QoS, Internet traffic analysis, and wireless and ad hoc networking.

Naoki Nakamura received the Dr. of Engineering degree in Information Science, from Tohoku University in 2008. He is now working as a Research Associate at Tohoku University School of Medicine, Japan. His research interests include wireless networking, distributed algorithms, and network performance evaluation.

Norio Shiratori received his doctoral degree from Tohoku University, Japan in 1977. Presently he is a Professor of the Research Institute of Electrical Communication, Tohoku University. He has engaged in research related to symbiotic computing paradigms between human and information technology. He was the recipient of IPSJ Memorial Prize Winning paper award in 1985, The Telecommunication Advancement Foundation Incorporation award in 1991 and many others. He was the vice president of IPSJ in 2002 and a fellow of IEEE, IPSJ and IEICE. 

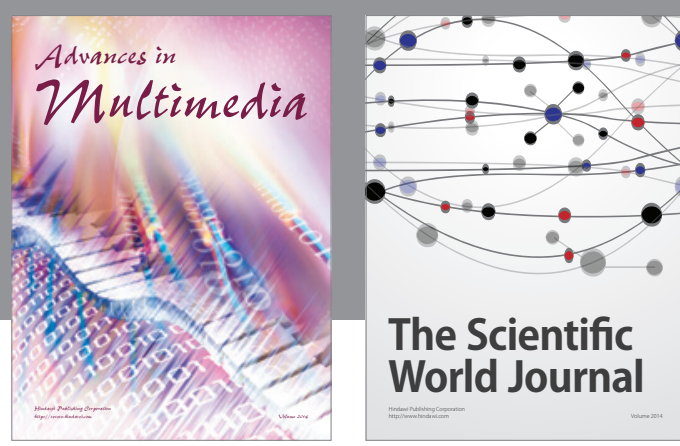

The Scientific World Journal
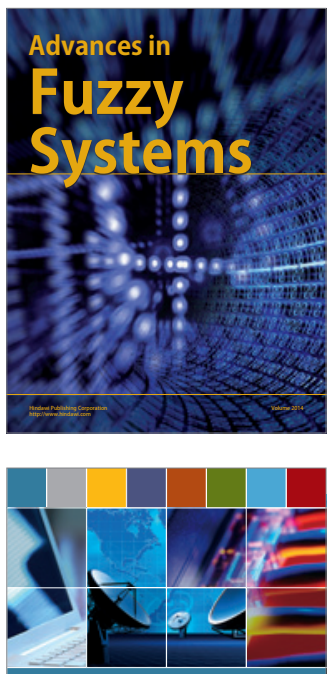

Computer Networks and Communications
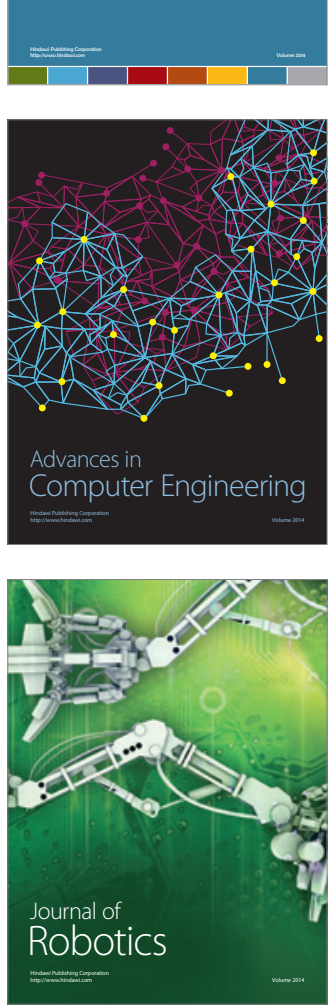
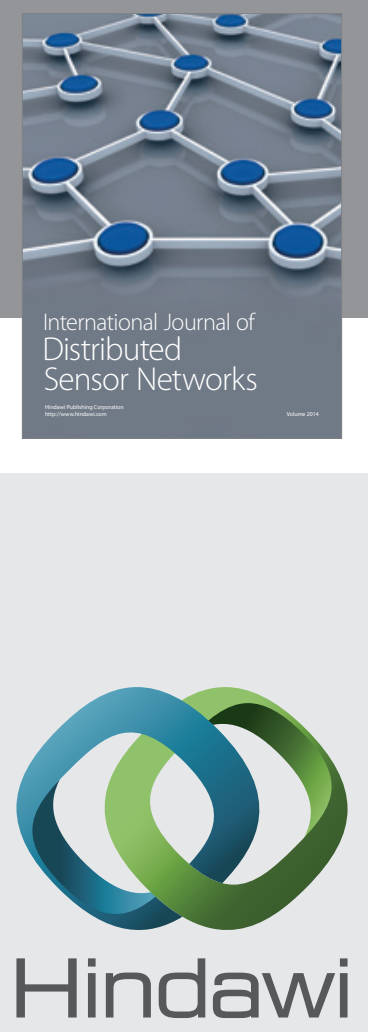

Submit your manuscripts at

http://www.hindawi.com
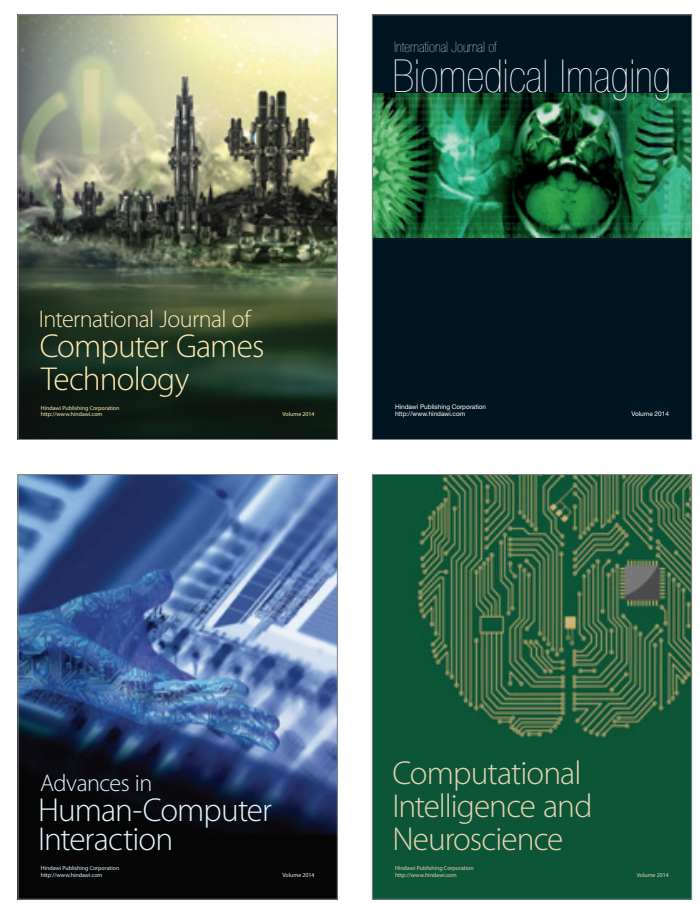
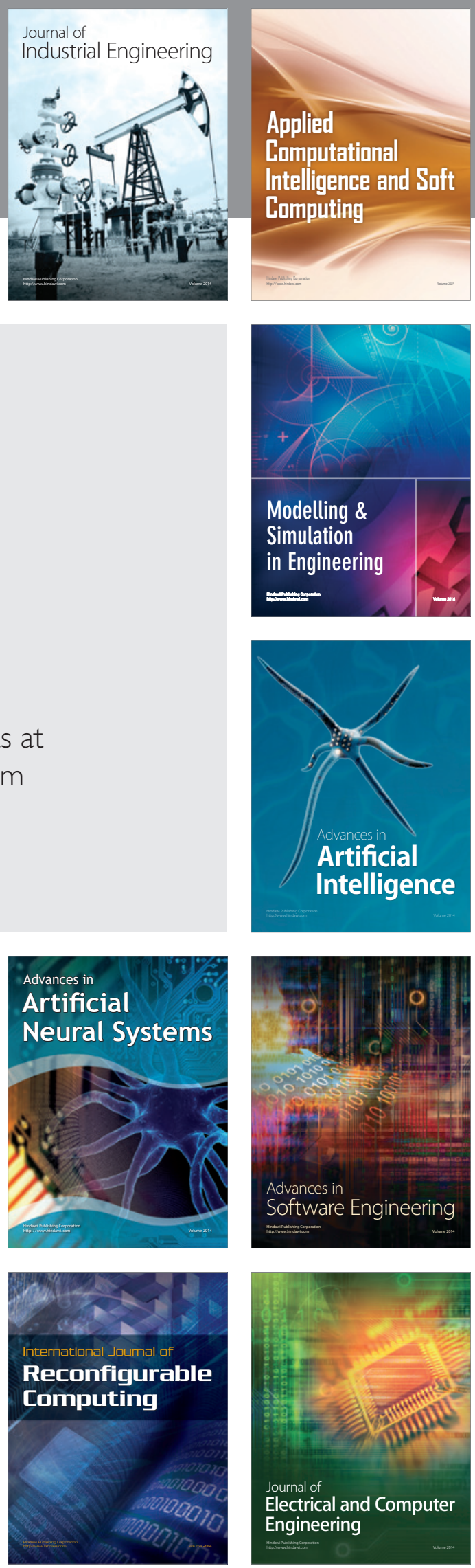